\title{
Pattern of Electrocardiographic and Echocardiographic Abnormalities Among HIV Patients in Port Harcourt, Nigeria
}

\author{
Ajala Aisha Oluwabunmi, Akpa Maclean Rumokere, Dodiyi-Manuel Sotonye
}

Department of Internal Medicine, University of Port Harcourt Teaching Hospital, Port-Harcourt, Nigeria

Email address:

ajalabunmi@gmail.com (A. A. Oluwabunmi)

\section{To cite this article:}

Ajala Aisha Oluwabunmi, Akpa Maclean Rumokere, Dodiyi-Manuel Sotonye. Pattern of Electrocardiographic and Echocardiographic Abnormalities Among HIV Patients in Port Harcourt, Nigeria. International Journal of HIV/AIDS Prevention, Education and Behavioural Science. Vol. 6, No. 1, 2020, pp. 15-24. doi: 10.11648/j.ijhpebs.20200601.13

Received: May 6, 2020; Accepted: May 29, 2020; Published: June 9, 2020

\begin{abstract}
Infection with HIV is independently associated with an increased risk of cardiovascular disease, while therapeutic use of HAART has been shown to increase the risk of metabolic derangements which may have potentially damaging effects on the cardiovascular system. The study was carried out to assess the pattern of electrocardiographic (ECG) and echocardiographic (ECHO) abnormalities among HAART experienced and HAART naïve HIV patients. This was a cross-sectional study of one hundred (100) HIV seropositive subjects and 100 age and sex matched controls to assess cardiovascular risk among HIV seropositive persons in Port-Harcourt, Rivers State, Nigeria. The results showed that the prevalence of ECG abnormalities among the cases was $49(49 \%)$ compared to $42(42 \%)$ of the controls $\left(\mathrm{c}^{2}=0.020, \mathrm{p}=0.886\right)$. Sinus tachycardia was the most common ECG abnormality in all study participants and was present in 37 (37\%) of the control subjects when compared to $21(21 \%)$ of the cases and this was statistically significant. T-wave inversion and prolonged QT interval were the next two most prevalent findings seen in $23 \%$ and $18 \%$ of the cases compared with $8 \%$ and $5 \%$ of the controls respectively and these were also statistically significant ( $\mathrm{p}=0.004$ and 0.003 respectively). Among the cases $10 \%$ had grade 1-2 diastolic dysfunctions compared with $23 \%$ of the controls and this was statistically significant $(\mathrm{p}=0.013)$. Ninety percent $(90 \%)$ however had normal diastolic function compared with $77 \%$ of the controls. Systolic dysfunction was found in only $2 \%$ of the HAART experienced cases compared to $10(10 \%)$ of the HAART naïve controls and this was also statistically significant $(\mathrm{p}=0.017)$. The study showed that electrocardiographic and echocardiographic abnormalities are common in HIV seropositive patients with ECG abnormalities being more common among the HAART treated subjects. These ECG \& ECHO abnormalities generally increase the cardiovascular risk profile of PLWHIV/AIDS and are independent predictors of $\mathrm{CV}$ mortality hence the need for prompt diagnosis and interventions.
\end{abstract}

Keywords: HIV, HAART, Electrocardiogram, Echocardiogram

\section{Introduction}

Cardiac manifestation of human immunodeficiency virus (HIV) and AIDS have become an increasingly important cause of morbidity and mortality. Cardiac involvement impacts on the natural history and prognosis of HIV disease. Patients living with HIV infection (PLWHIV) can have a variety of cardiovascular manifestation including myocarditis, pericarditis, cardiomyopathy, pulmonary vascular disease, pulmonary hypertension and vascular diseases including coronary artery disease [1]. An increased prevalence of cardiovascular diseases (CVD) in People living with HIV (PLWHIV) has been reported over the years and this has been attributed to increase prevalence of traditional risk factors and also by an increase in the prevalence of dyslipidaemia, and also vascular changes conferred by the effect of antiretroviral agents and also by the virus itself [2, 3, 4]. In the current era of effective Highly Active Antiretroviral Therapy (HAART) use, cardiovascular risk has emerged as a significant marker of morbidity and mortality among surviving HIV-positive patients. It is questionable whether HIV itself confers a significant cardiovascular risk, as some studies have shown conflicting results [3]. Studies supports the collaborative effect of a triad of the virus, immune reconstitution and HAART use, which initiates premature endothelial activation and may lead to the structural 
composition of arterial lesions in HIV patients [3, 5]. The availability of HAART has dampened the effect of opportunistic infections and has promoted longevity in HIV patients. However, this has resulted in PLWHIV becoming increasingly prone to cardiovascular (CV) manifestations, particularly premature atherosclerosis not associated with the conventional predisposing risk factors that are emphasized in HAART-naïve patients. The electrocardiogram, which is fairly widely available in resource-poor countries like Nigeria is helpful in evaluating abnormalities in HIV/AIDS patients whether or not they were suspected of having cardiac disease. Several studies have reported an increased prevalence of ECG abnormalities in HIV patients including those on HAART. These findings range from minor to major ECG abnormalities and are as a result of an increase in cardiac abnormalities in PLWHIV/AIDS such as cardiomyopathy, myocarditis, pericarditis, pulmonary hypertension [5]. The association of HIV infection and cardiac pathology has been recognized since the early stages of the epidemic. Post-mortem studies have shown an incidence of AIDS-related heart disease higher than that diagnosed clinically ante mortem [4]. It is therefore possible that many patients have cardiac abnormalities that are not recognized during the course of their illness. These abnormalities have been attributed directly or indirectly to the HIV virus and/or side effects of antiretroviral therapy. Determining the deleterious effects of HIV may prove echocardiography to be a useful non-invasive, radiation free tool to assess cardiac function and monitor for cardiac abnormalities in HIV patients. Infection with HIV is independently associated with an increased risk of cardiovascular disease [5]. Furthermore, the use of HAART has been shown to cause several metabolic derangements, which may have potentially damaging effects on the cardiovascular system. Assessment of $\mathrm{CV}$ risk in HIV seropositive patients is therefore warranted $[5,6]$. The study was carried out to assess the pattern of electrocardiographic and echocardiographic abnormalities among HAART experienced and HAART naïve HIV patients in Port Harcourt, Rivers State, Nigeria.

\section{Methodology}

\subsection{Study Design}

The study was a cross-sectional study. Cases were defined as HIV-seropositive patients who had been on HAART for 12 months and above while the controls were age and sex matched HIV-seropositive HAART naïve patients.

\subsection{Study Setting}

The study was carried out at the Anti-Retroviral clinic of the University of Port Harcourt Teaching Hospital, Rivers State, Nigeria. The hospital is a tertiary hospital located in the city of Port-Harcourt and serves as a referral medical centre for neighbouring states such as Bayelsa, Delta and Abia in Southern Nigeria.

\subsection{Ethical Consideration}

Ethical approval for this study was obtained from the Ethics and Research Committee of the University of PortHarcourt Teaching Hospital before commencement of the study. Willing informed written consent was obtained from the patients before recruitment into the study. Subjects found to have abnormalities were advised and referred appropriately for further management. Personal data of the subjects remained confidential.

\subsection{Study Sample and Sampling}

One hundred previously diagnosed HIV seropositive patients who have been on HAART for 12 months and above presenting at the Anti-retroviral clinic of the University of Port-Harcourt Teaching Hospital were recruited into the study after meeting the eligibility criteria set as follows:

1. Individuals aged above 18 years

2. Individuals that have confirmed diagnosis of HIV seropositive,

3. Individuals that are on HAART for $\geq 12$ months

For each case recruited into the study, a control of same sex and similar age (+/-2 years) was selected recruited into the study after meeting the eligibility criteria as stated below:

1. Individuals aged above 18 years,

2. Individuals that have been confirmed HIV sero-positive and HAART naïve.

3. Individuals with no pre-existing illnesses.

\subsection{Data Collection}

A structured interviewer-administered questionnaire was used to collect demographic information and disease related variables from the subjects. A detailed physical examination was conducted to determine weight, height, abdominal circumference and blood pressure.

\subsection{Electrocardiogram}

A resting ECG was done using a 3-channel portable 12-lead ECG machine, Contec ${ }^{\circledR}$ ECG100G in the ECG laboratory in the standard fashion. The ECG parameters including LVH, left or right atrial abnormalities, rhythm disturbances, QT interval prolongation, ST-T abnormalities and presence of pathological $Q$ waves were assessed and reported according to the Minnesota code for interpretation of ECG abnormalities. An abnormal ECG was defined by presence of left ventricular hypertrophy using the Sokolow Lyon's criteria, arrhythmias, AV conduction defects, QT prolongation, ST segment and T waves changes, $\mathrm{P}$ wave abnormalities, abnormal $\mathrm{Q}$ wave and abnormal axis deviation [7].

\subsection{Echocardiogram}

Echocardiography was performed using a Mindray ${ }^{\circledR}$ ultrasound machine model DC-N6 equipped with $2.5 \mathrm{MHz}$ transducer. Each subject was briefed on the non-invasive nature of the procedure to allay fear and anxiety. Twodimensional (2D), M-mode, pulse-wave, continuous wave and 
colour Doppler echocardiography assessment was done with the subject in left lateral decubitus position and targeted echocardiographic estimations were taken using the internal analysis software of the machine. Two-Dimensional Oriented Motion-mode measurements of left atrial diameter (LAD), aortic size (AO), interventricular septal thickness in diastole (IVSd), left posterior wall thickness in diastole (LVPWd), and left ventricular end diastolic diameter (LVEDd) just beyond the tips of the mitral valve leaflets were obtained from the parasternal long axis view according to the American Society of Echocardiography Guidelines [8]. Left ventricular mass in grams was calculated using the American Society of Echocardiography formula modified by Devereux.

$$
\begin{gathered}
\mathrm{LVM}(\mathrm{gm})=0.8 \times\left[1 . 0 4 \left(\mathrm{LVIDd}+\mathrm{PWDd}+\mathrm{IVSDd}^{3}-\right.\right. \\
\left.\mathrm{LVIDd}^{3}\right]+0.6 \mathrm{~g}
\end{gathered}
$$

Left ventricular mass was indexed to body surface area using cut-off values of $115 \mathrm{~g} / \mathrm{m}^{2}$ and $95 \mathrm{~g} / \mathrm{m}^{2}$ for men and women respectively. Relative wall thickness was also calculated as $2 \times$ posterior wall thickness in end diastole divided by LV end diastolic diameter. A partition value of 0.42 for RWT was used for both men and women. Patients with increased LV mass index and increased RWT were considered to have concentric hypertrophy, and those with increased LV mass index and normal RWT were considered to have eccentric hypertrophy. Those with normal LV mass index and increased or normal RWT were considered to have concentric remodeling or normal geometry respectively [5] LV systolic function was assessed using ejection fraction (EF) and fractional shortening (FS). Theses was calculated by the machine using the Teicholz formula [9]

The normal values for fractional shortening was taken as $27-45 \%$ for males and $25-43 \%$ for females while the normal values for ejection fraction was taken as $\geq 55 \%, 45-54 \%$ as mildly depressed ejection fraction, $35-44 \%$ as moderately depressed ejection fraction and $\leq 35 \%$ as severely depressed ejection fraction [1-3] Left ventricular diastolic pattern was assessed by Echo pulsed Doppler analysis according to the American Society of Echocardiography and European
Association of Cardiovascular Imaging Guidelines [10]. The diastolic mitral flow assessed by early diastolic peak velocity (E), the ratio of $\mathrm{E}$ to $\mathrm{A}(\mathrm{E} / \mathrm{A})$ and the deceleration time of the early mitral velocity were recorded with the sample volume at the mitral leaflet tips.

Pulse Doppler recordings of transmitral flow velocities were obtained between the tips of the mitral leaflets for measuring peak early left ventricular filling velocity/peak atrial filling velocity (E/A) and deceleration time (DecT). Valsalva maneuver was performed when applicable. Deceleration time was measured as the time from peak $\mathrm{E}$ velocity to the time when the $\mathrm{E}$ wave descent intercepts the zero line. Isovolumic relaxation time was measured with a pulsed wave Doppler beam intersecting left ventricular outflow and inflow tract. Tissue Doppler imaging (TDI) was done with the pulse wave Doppler on the septal and lateral mitral annulus and the peak early diastolic velocities measured (E'). An average E' is calculated and also the E/E' was also measured [11]

\subsection{Data Analysis}

All data were analyzed using the Statistical Package for Social Sciences (SPSS) version 20 analytic software. Continuous variables were analyzed using independent sample t-test and results expressed as mean \pm standard deviation, while the pattern of electrocardiographic and echocardiographic abnormalities was analyzed using chisquare test or two tailed Fisher's exact and results expressed as frequency counts and percentages. All tests were considered to be statistically significant at the p-value $<0.05$.

\section{Results}

There was no significant difference in the mean age of the cases and the controls: $37.28 \pm 8.92$ (range 18-57 years) versus $36.20 \pm 8.92$ years (range 19-58 years). The most common age groups among both the cases and controls were the 20-29 years and 30-39 years as shown in Table 1 .

\begin{tabular}{|c|c|c|c|c|}
\hline Variable & Case $n=100(\%)$ & Control n=100 (\%) & Total n=200 (\%) & Chi-square (p-value) \\
\hline \multicolumn{5}{|l|}{ SEX } \\
\hline Female & $65(65.0)$ & $61(61.0)$ & $74(37.0)$ & \multirow[t]{2}{*}{$0.558(0.343)$} \\
\hline Male & $35(35.0)$ & $39(39.0)$ & $126(63.0)$ & \\
\hline \multicolumn{4}{|l|}{ AGE GROUP } & \multirow{6}{*}{$6.993(0.136)$} \\
\hline$<20$ years & $1(1.0)$ & $2(2.0)$ & $3(1.5)$ & \\
\hline 20-29 years & $25(25.0)$ & $22(22.0)$ & $47(23.5)$ & \\
\hline $30-39$ years & $41(47.0)$ & $47(41.0)$ & $88(44.0)$ & \\
\hline 40-49 years & $23(23.0)$ & $20(20.0)$ & $43(21.5)$ & \\
\hline$\geq 50$ years & $10(10.0)$ & $9(9.0)$ & $19(9.5)$ & \\
\hline \multicolumn{4}{|c|}{ EDUCATIONAL STATUS } & \multirow{5}{*}{$* *(0.745)$} \\
\hline None & $0(0.0)$ & $1(1.0)$ & $1(0.5)$ & \\
\hline Primary & $20(20.0)$ & $18(18.0)$ & $38(19.0)$ & \\
\hline Secondary & $47(47.0)$ & $52(52.0)$ & $99(49.5)$ & \\
\hline Tertiary & $33(33.0)$ & $29(29.0)$ & $62(31.0)$ & \\
\hline \multirow{2}{*}{$\begin{array}{l}\text { MARITAL STATUS } \\
\text { Single }\end{array}$} & & & & \multirow{2}{*}{$* *(0.354)$} \\
\hline & $34(34.0)$ & $45(45.0)$ & $79(39.5)$ & \\
\hline
\end{tabular}

Table 1. Socio-demographic characteristics of Study Subjects. 


\begin{tabular}{lllll}
\hline Variable & Case $\mathbf{n = 1 0 0}(\mathbf{\%})$ & Control $\mathbf{n = 1 0 0}(\mathbf{\%})$ & Total $\mathbf{n = 2 0 0}(\mathbf{\%})$ & Chi-square (p-value) \\
\hline Married & $61(61.0)$ & $49(49.0)$ & $110(55.0)$ & \\
Separated & $1(1.0)$ & $1(1.0)$ & $2(1.0)$ & \\
Divorce & $4(4.0)$ & $5(5.0)$ & $9(4.5)$ & \\
\hline
\end{tabular}

*Statistically significant ** Fishers exact.

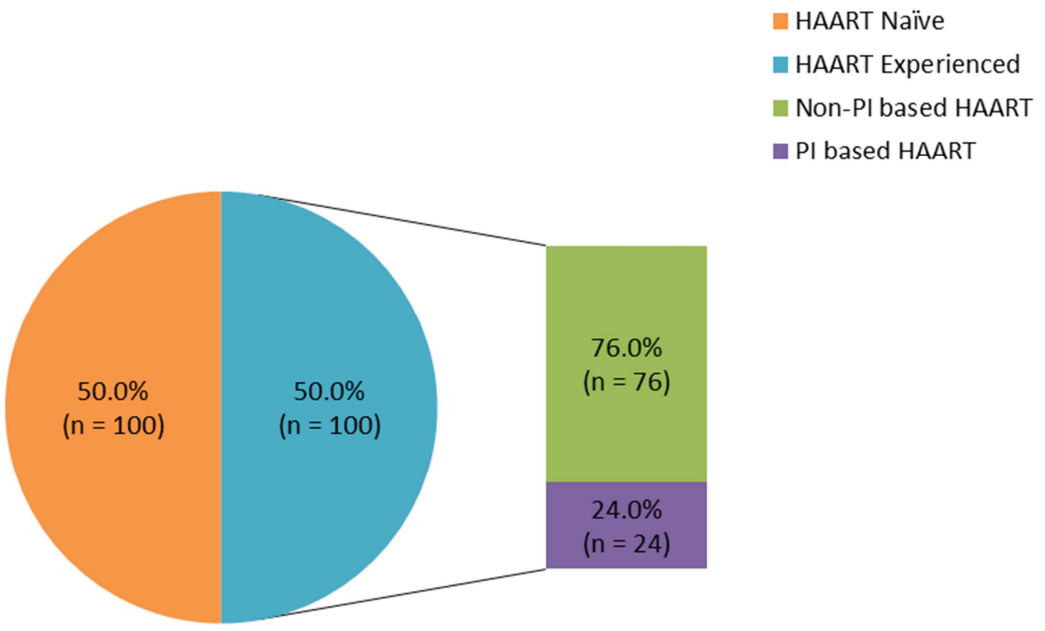

Figure 1. Type of HAART among Study subjects.

Figure 1 shows the distribution of the HARRT regimen among the subjects, the number of cases on a non-PI based HAART was $76(76 \%)$, while $24(24 \%)$ were on PI-based HAART.

Table 2 shows ECG abnormalities among the cases and controls.

As shown in table 2, The prevalence of ECG abnormalities among the cases was 49 (49\%) compared to $42(42 \%)$ of the controls $\left(x^{2}=0.020, \mathrm{p}=0.886\right)$ Sinus tachycardia was the commonest ECG abnormality in all study groups and was present in $37(37 \%)$ of the control subjects when compared to $21(21 \%)$ of the cases and this was statistically significant. $\mathrm{T}$-wave inversion and prolonged QT interval were the next two most prevalent findings seen in $23 \%$ and $18 \%$ of the cases compared with $8 \%$ and $5 \%$ of the controls respectively and these were also statistically significant $(p=0.004$ and 0.003 respectively). There was however no significant difference in the prevalence of LVH. Other ECG findings reported are incomplete bundle branch block, early repolarization abnormalities, ventricular extra systole (VES), ST-segment elevation/depression, pathological Q waves, left axis deviation, left atrial enlargement and first-degree atrioventricular block (AV block).

ECG abnormalities were more prevalent in the non-PI based HAART group when compared with the PI-based HAART group. Left ventricular hypertrophy (LVH) was seen more in the non-PI based HAART group and this difference was statistically significant. Other findings such as T-wave inversion, QT prolongation, and early repolarization were more in the non-PI based group but with no statistical significant difference. None of the patients on a PI-based HAART group had ventricular extra systole and first-degree AV block as shown in Table 3 .

Table 2. Prevalence of ECG abnormalities among cases and controls.

\begin{tabular}{|c|c|c|c|c|}
\hline Variable & $\begin{array}{l}\text { Cases } \\
n=100(\%)\end{array}$ & $\begin{array}{l}\text { Control } \\
n=100(\%)\end{array}$ & $\begin{array}{l}\text { Total } \\
\text { n=200 (\%) }\end{array}$ & $\begin{array}{l}\text { Chi-square } \\
\text { (p value) }\end{array}$ \\
\hline Sinus Tachycardia & $21(21.0)$ & $37(37.0)$ & $58(29.0)$ & $6.217(0.013)^{*}$ \\
\hline T-wave inversion & $23(23.0)$ & $8(8.0)$ & $31(15.5)$ & $8.303(0.004)^{*}$ \\
\hline QT prolongation & $18(18.0)$ & $5(5.0)$ & $23(11.5)$ & $8.589(0.003)^{*}$ \\
\hline LVH & $22(22.0)$ & $15(15.0)$ & $37(18.5)$ & $1.625(0.202)$ \\
\hline Incomplete RBBB & $3(3.0)$ & $6(6.0)$ & $9(4.5)$ & $* *(0.498)$ \\
\hline Early repolarization & $10(10.0)$ & $5(5.0)$ & $15(7.5)$ & $2.446(0.118)$ \\
\hline ST segment depression & $2(2.0)$ & $0(0.0)$ & $2(1.0)$ & $3.436(0.064)$ \\
\hline VES & $2(2.0)$ & $5(5.0)$ & $7(3.5)$ & $1.332(0.248)$ \\
\hline Pathological Q waves & $1(1.0)$ & $1(1.0)$ & $2(1.0)$ & $* *(1.000)$ \\
\hline First degree AV block & $2(2.0)$ & $0(0.0)$ & $2(1.0)$ & $3.437(0.064)$ \\
\hline Left atrial enlargement & $5(5.0)$ & $2(2.0)$ & $7(3.5)$ & $1.332(0.248)$ \\
\hline Left axis deviation & $6(6.0)$ & $2(2.0)$ & $8(4.0)$ & $2.083(0.149)$ \\
\hline
\end{tabular}

*Statistically significant; **Fishers exact. Key: VES=ventricular extrasystole; AV=atrioventricular; LVH=left ventricular hypertrophy; RBBB=right bundle branch block. 
Table 3. Comparison of ECG abnormalities by type of Antiretroviral Therapy (ART) among HIV subjects.

\begin{tabular}{lllll}
\hline Variable & Non-PI based $\mathbf{n = 7 6}(\mathbf{\%})$ & PI-based $\mathbf{n = 2 4}(\mathbf{\%})$ & Total $\mathbf{n = 1 0 0}(\mathbf{\%})$ & Chi-square/t-test $(\mathbf{p}$ value) \\
\hline Sinus Tachycardia & $14(66.7)$ & $7(33.3)$ & $21(100.0)$ & $1.270(0.260)$ \\
T-wave inversion & $21(91.3)$ & $2(8.7)$ & $23(100.0)$ & $3.836(0.050)$ \\
QT prolongation & $13(72.2)$ & $5(27.8)$ & $18(100.0)$ & $0.172(0.679)$ \\
LVH & $13(59.1)$ & $9(40.9)$ & $22(100.0)$ & $4.421(0.035)^{*}$ \\
Incomplete RBBB & $0(0.0)$ & $3(100.0)$ & $3(100.0)$ & $* *(0.561)$ \\
Early repolarization & $6(60.0)$ & $4(40.0)$ & $10(100.0)$ & $* *(0.246)$ \\
ST segment depression & $1(50.0)$ & $1(50.0)$ & $2(100.0)$ & $* *(0.424)$ \\
VES & $2(2.0)$ & $0100.0)$ & $2(100.0)$ & $* *(1.000)$ \\
Pathological Q waves & $0(0.0)$ & $1(100.0)$ & $1(100.0)$ & $* *(0.270)$ \\
First degree AV block & $2(100.0)$ & $0(100.0)$ & $2(100.0)$ & $* *(1.000)$ \\
Left atrial enlargement & $3(60.0)$ & $2(40.0)$ & $5(100.0)$ & $* *(0.591)$ \\
Left axis deviation & $3(50.0)$ & $3(50.0)$ & $6(100.0)$ & $* *(0.148)$ \\
\hline
\end{tabular}

*Statistically significant; **Fishers Exact. Key: VES=ventricular extrasystole, AV=atrioventricular, $\mathrm{LVH}=$ left ventricular hypertrophy, RBBB=right bundle branch block.

Table 4. Mean echocardiographic parameters in Cases and Controls.

\begin{tabular}{llll}
\hline Parameters & Cases n=100 & Controls n=100 & Student t-test (P value) \\
\hline LAD $(\mathrm{cm})$ & $3.45 \pm 0.42$ & $3.43 \pm 0.42$ & $0.402(0.688)$ \\
AoD $(\mathrm{cm})$ & $2.49 \pm 0.34$ & $2.54 \pm 0.41$ & $0.959(0.339)$ \\
IVSd $(\mathrm{cm})$ & $0.92 \pm 0.23$ & $0.94 \pm 0.26$ & $0.631(0.529)$ \\
LVIDd $(\mathrm{cm})$ & $4.87 \pm 0.51$ & $4.76 \pm 0.55$ & $1.542(0.125)$ \\
LVPWd (cm) & $0.96 \pm 0.17$ & $0.97 \pm 0.18$ & $0.085(0.932)$ \\
LVM $(\mathrm{g})$ & $164.37 \pm 54.37$ & $160.10 \pm 48.69$ & $0.585(0.559)$ \\
LVMI $\left(\mathrm{g} / \mathrm{m}^{2}\right)$ & $97.3 \pm 31.22$ & $95.1 \pm 27.41$ & $0.530(0.597)$ \\
RWT & $0.40 \pm 0.08$ & $0.41 \pm 0.09$ & $0.914(0.362)$ \\
SV $(\mathrm{ml})$ & $80.42 \pm 20.71$ & $77.90 \pm 20.32$ & $0.872(0.384)$ \\
LVEF $(\%)$ & $71.99 \pm 7.11$ & $68.99 \pm 8.73$ & $2.676(0.008)^{*}$ \\
LVFS $(\%)$ & $41.70 \pm 6.32$ & $39.06 \pm 7.20$ & $2.777(0.006)^{*}$ \\
E (ms) & $73.94 \pm 17.14$ & $71.8 \pm 18.91$ & $0.856(0.393)$ \\
A (ms) & $51.69 \pm 15.40$ & $52.56 \pm 15.22$ & $0.489(0.676)$ \\
E/A ratio & $1.54 \pm 0.61$ & $1.47 \pm 0.52$ & $0.935(0.351)$ \\
Dec T (ms) & $169.0 \pm 31.8$ & $171.0 \pm 24.28$ & $0.498(0.619)$ \\
IVRT (s) & $82.94 \pm 16.44$ & $83.17 \pm 18.34$ & $0.074(0.072)$ \\
TAPSE (cm) & $2.22 \pm 0.35$ & $2.13 \pm 0.48$ & $1.551(0.123)$ \\
PVAccT (ms) & $125.56 \pm 25.53$ & $124.75 \pm 30.77$ & $0.203(0.840)$ \\
\hline
\end{tabular}

Values are mean \pm SD. *statistically significant. Key: $\mathrm{LAD}=$ left atrial diameter; $\mathrm{AOD}=$ aortic root diameter; IVSd=interventricular septal thickness in diastole; LVIDd=left ventricular internal diameter in diastole; LVPWd=left ventricular posterior wall thickness in diastole; LVM=left ventricular mass; LVMI=left ventricular mass indexed to body surface area; RWT=relative wall thickness; TAPSE=tricuspid annular plane systolic excursion. SV=stroke volume; LVEF=left ventricular ejection fraction; $L V F S=l$ eft ventricular fractional shortening; $E=$ early diastolic filling; $A=$ atrial contraction; $E / A=$ ratio of early (E) to late (A) diastolic filling velocities in mitral inflow; DecT=deceleration, IVRT=isovolumic relaxation time.

Table 5. Comparison of ECHO parameters based on type of HAART used among cases.

\begin{tabular}{llll}
\hline Parameters & Non-PI based $(\mathbf{n}=\mathbf{7 6})$ & PI-based $(\mathbf{n}=\mathbf{2 4})$ & t-test $(\mathbf{P}$ value) \\
\hline Age & $37.7 \pm 9.0$ & $36.0 \pm 8.7$ & $0.805(0.423)$ \\
HAART duration $(\mathrm{mos})$ & $42.5 \pm 19.9$ & $111.4 \pm 16.6$ & $16.921(0.0001)^{*}$ \\
Weight $(\mathrm{kg})$ & $62.04 \pm 8.76$ & $64.92 \pm 11.0$ & $1.318(0.191)$ \\
BMI $\left(\mathrm{kg} / \mathrm{m}^{2}\right)$ & $23.76 \pm 2.93$ & $24.01 \pm 3.54$ & $0.343(0.733)$ \\
SBP $(\mathrm{mmHg})$ & $129.72 \pm 13.91$ & $136.04 \pm 13.83$ & $1.942(0.055)$ \\
DBP $(\mathrm{mmHg})$ & $82.37 \pm 5.85$ & $83.46 \pm 7.18$ & $0.752(0.454)$ \\
LAD $(\mathrm{cm})$ & $3.42 \pm 0.44$ & $3.56 \pm 0.31$ & $1.490(0.139)$ \\
AoD $(\mathrm{cm})$ & $2.49 \pm 0.36$ & $2.49 \pm 0.31$ & $0.025(0.980)$ \\
IVSd $(\mathrm{cm})$ & $0.91 \pm 0.23$ & $0.97 \pm 0.19$ & $1.178(0.242)$ \\
LVIDd $(\mathrm{cm})$ & $4.88 \pm 0.52$ & $4.83 \pm 0.49$ & $0.527(0.600)$ \\
LVPWd $(\mathrm{cm})$ & $0.94 \pm 0.17$ & $1.03 \pm 0.17$ & $2.287(0.024)^{*}$ \\
LVM $(\mathrm{g})$ & $161.67 \pm 58.0$ & $172.9 \pm 40.51$ & $0.882(0.380)$ \\
LVMI $\left(\mathrm{g} / \mathrm{m}^{2}\right)$ & $96.29 \pm 21.32$ & $97.57 \pm 33.83$ & $0.174(0.863)$ \\
RWT & $0.39 \pm 0.08$ & $0.43 \pm 0.10$ & $2.215(0.029)$ \\
\hline
\end{tabular}




\begin{tabular}{llll}
\hline Parameters & Non-PI based $(\mathbf{n}=\mathbf{7 6})$ & PI-based $(\mathbf{n}=\mathbf{2 4})$ & t-test $(\mathbf{P}$ value) \\
\hline SV $(\mathrm{ml})$ & $79.46 \pm 21.21$ & $83.48 \pm 19.33$ & $0.827(0.410)$ \\
LVEF $(\%)$ & $71.30 \pm 7.14$ & $74.17 \pm 6.70$ & $1.744(0.084)$ \\
LVFS $(\%)$ & $41.16 \pm 6.32$ & $43.40 \pm 6.24$ & $1.532(0.129)$ \\
E $(\mathrm{ms})$ & $72.96 \pm 16.03$ & $77.04 \pm 20.35$ & $1.017(0.312)$ \\
A $(\mathrm{ms})$ & $50.96 \pm 15.02$ & $53.97 \pm 16.61$ & $0.837(0.405)$ \\
E/A ratio & $1.52 \pm 0.60$ & $1.61 \pm 0.65$ & $0.649(0.518)$ \\
Dec T (ms) & $165.61 \pm 28.54$ & $179.75 \pm 39.32$ & $1.927(0.057)$ \\
IVRT $(\mathrm{s})$ & $82.87 \pm 15.90$ & $83.17 \pm 18.34$ & $0.077(0.938)$ \\
TAPSE $(\mathrm{cm})$ & $2.21 \pm 0.36$ & $2.25 \pm 0.31$ & $0.472(0.638)$ \\
PVAccT $(\mathrm{ms})$ & $123.41 \pm 30.38$ & $129.0 \pm 32.28$ & $0.774(0.441)$ \\
\hline
\end{tabular}

Values are mean $\pm \mathrm{SD} .{ }^{*}$ statistically significant. Key: $\mathrm{LAD}=$ left atrial diameter; aortic root diameter; IVSd=interventricular septal thickness in diastole; LVIDd=left ventricular internal diameter in diastole; LVPWd=left ventricular posterior wall thickness in diastole; LVM=left ventricular mass; LVMI=left ventricular mass indexed to body surface area; RWT=relative wall thickness; TAPSE=tricuspid annular plane systolic excursion. SV=stroke volume; $\mathrm{LVEF}=$ left ventricular ejection fraction; $\mathrm{LVFS}=$ left ventricular fractional shortening; $\mathrm{E}=$ early diastolic filling; $\mathrm{A}=$ atrial contraction; $\mathrm{E} / \mathrm{A}=$ ratio of early (E) to late (A) diastolic filling velocities in mitral inflow; DecT=deceleration, IVRT=isovolumic relaxation time; mos=months.

As shown in Tables $4 \& 5$, the control subjects had a slightly thicker interventricular septal diameter in diastole (IVSd) than the cases, there was no significant difference in the mean left ventricular internal diameter in diastole (LVIDd) and mean left ventricular posterior wall thickness in diastole (LVPWd) in the cases and controls. There was however a significant difference in the mean LVPWd in the PI-based group when compared with the non-PI-based group $(p=0.024)$. There was no significant difference between the mean left ventricular mass (LVM) of the cases and controls $(p=0.559)$. Among the PI based HAART subjects, the mean LVM was which higher than in the non-PI based HAART group but this was also not statistically significant. There was no significant difference in the mean left ventricular mass index (LVMI) and relative wall thickness (RWT) of the cases and control subjects ( $\mathrm{p}=0.597$ and $\mathrm{p}=0.362$ respectively). The male subjects had a significantly higher LVMI than the female subjects $107.03 \pm 28.5$ versus $89.85 \pm 28.1$. ( $\mathrm{t}=4.150$, $\mathrm{p}=<0.001$ ). Among the cases the PI based HAART subjects had a higher LVMI than the non-PI based HAART but this was not statistically significant $(p=0.863)$. The RWT was however significantly higher in the PI-based HAART group $(p=0.029)$. The mean left atrial diameter (LAD) was higher among the cases than the controls but this was not significant $(\mathrm{p}=0.688)$. The aortic root diameter was however higher among the control subjects but with no significant difference. The PI-based HAART had a non-significantly higher mean LAD than the non PI-based group with an equal mean aortic root diameter $(\mathrm{p}=0.139$ and $\mathrm{p}=0.980)$. Twelve subjects in this study had a low left ventricular systolic function assessed with the ejection fraction (EF) and fractional shortening (FS). Ten subjects $(10 \%)$ were HAART naive controls while the remaining two (2\%) were case subjects. The mean EF and FS were lower in the control subjects compared to the case subjects and this difference was statistically significant ( $\mathrm{p}=0.008$ and $\mathrm{p}=0.006$ respectively). The PI-based HAART group had a higher mean EF and FS than the non PI-based HAART subjects but this was not statistically significant ( $p=0.084$ and 0.129 respectively). The diastolic function of the left ventricle was assessed using the mitral inflow velocity and tissue Doppler. The mitral inflow velocity was divided into: E/A Ratio (E/A); the mean E/A ratio for both the cases and controls, the PI-based and non-PI based HAART subjects were not statistically significant. Deceleration Time (DT): There was no statistically significant difference in the mean DT between cases and control group. The mean DT of the PI-based HAART group compared to the non-PI based group was also not significantly different. Isovolumic Relaxation Time (IVRT): The control group had a shorter mean IVRT of compared to the case subjects but this was not statistically significant. The mean IVRT between the PI based and Non-PI based HAART group was also not statistically significant. Mitral E/Tissue Doppler E' Ratio (E/E'): The mean E/E' ratio for the case subjects was significantly higher than the controls. There was however no significant difference in the mean $\mathrm{E} / \mathrm{E}$ ' ratio in the treatment arm on a PI-based compared with the non-PI based HAART subjects.

The mean TAPSE was not significantly higher among the cases when compared to the controls $(\mathrm{p}=0.123)$. The PI-based HAART subjects however had a slightly higher but nonsignificant mean TAPSE value than the non-PI based HAART subjects. There was no significant difference in the mean PVAccT in the cases when compared to the controls. Ten (10\%) of the cases had pulmonary hypertension $(\mathrm{PH})$ compared to 5 $(5 \%)$ of the controls $(\mathrm{p}=0.179)$. The overall prevalence of $\mathrm{PH}$ was $7.5 \%$. There was also no significant difference when the PIbased and non-PI based HAART groups were compared.

The diastolic function observed in the study population is as follow; among the cases ninety (90\%) had normal diastolic function compared with $77 \%$ of the controls, while $10 \%$ of the cases had grade 1-2 diastolic dysfunction compared with $23 \%$ of the controls and this was statistically significant $(p=0.013)$. Systolic dysfunction was found in only $2 \%$ of the cases compared to $10(10 \%)$ of the controls and this was also statistically significant $(\mathrm{p}=0.017)$. There was however no significant difference in the prevalence of pericardial effusion, pulmonary hypertension and dilated cardiomyopathy as shown in Table 6. 
Table 6. Echocardiographic abnormalities among cases and controls.

\begin{tabular}{lllll}
\hline Echocardiographic Abnormalities & Cases n=100 & Controls n=100 & Total n=200 & Chi-square (P value) \\
\hline LVH & & & & \\
Yes & $31(31.0)$ & $29(29.0)$ & $60(30.0)$ & $2.845(0.416)$ \\
No & $69(69.0)$ & $71(71.0)$ & $140(70.0)$ & \\
$\begin{array}{l}\text { Diastolic dysfunction } \\
\text { Yes }\end{array}$ & $10(10.0)$ & $23(23.0)$ & $33(16.5)$ & $6.133(0.013)^{*}$ \\
No & $90(90.0)$ & $77(77.0)$ & $167(83.5)$ & \\
Systolic dysfunction & & & & $5.674(0.017)^{*}$ \\
Yes & $2(2.0)$ & $10(10.0)$ & $12(6.0)$ & \\
No & $98(98.0)$ & $90(90.0)$ & $188(94.0)$ & $0.523(0.469)$ \\
Pericardial effusion & & & & \\
Yes & $8(8.0)$ & $11(11.0)$ & $19(9.5)$ & $1.802(0.179)$ \\
No & $92(92.0)$ & $89(89.0)$ & $181(90.5)$ & \\
Pulmonary hypertension & $10(10.0)$ & $5(5.0)$ & $15(7.5)$ & $2.020(0.155)$ \\
Yes & $90(90.0)$ & $95(95.0)$ & $185(92.5)$ & \\
No & & & $2(1.0)$ & \\
Dilated cardiomyopathy & $0(0.0)$ & $2(2.0)$ & $198(99.0)$ & \\
Yes & $100(100.0)$ & $98(98.0)$ & & \\
No & & & & \\
\hline
\end{tabular}

*statistically significant. LVH=left ventricular hypertrophy.

As shown in table 7, all the cases with diastolic dysfunction were on a non-PI based HAART but this was not statistically significant $(\mathrm{p}=0.061)$. More patients on a non-PIbased HAART had LVH when compared to the PI-based HAART group but this was also not statistically significant $(\mathrm{p}=0.060)$. Systolic dysfunction was found in one subject from each HAART group. Pericardial effusion and pulmonary hypertension were seen more among the non-PI based HAART group but this was also not statistically significant. $(\mathrm{p}=0.427$ and 0.640 respectively).

Table 7. Echocardiographic abnormalities based on type of ART among HIV subjects.

\begin{tabular}{lllll}
\hline Echocardiographic abnormalities & Non-PI based $\mathbf{n = 7 6}(\mathbf{\%})$ & PI-based $\mathbf{n = 2 4}(\mathbf{\%})$ & Total $\mathbf{n}=\mathbf{1 0 0}$ & Chi-square (P value) \\
\hline LVH & $21(27.6)$ & $10(41.7)$ & $31(31.0)$ & $7.395(0.060)$ \\
Yes & $55(72.4)$ & $14(58.3)$ & $69(69.0)$ & \\
No & $10(13.2)$ & $0(0.0)$ & $10(10.0)$ & $3.509(0.061)$ \\
Diastolic dysfunction & $66(86.8)$ & $24(100.0)$ & $90(90.0)$ & \\
Yes & $1(1.3)$ & $1(4.2)$ & $2(2.0)$ & $0.756(0.384)$ \\
No & $75(98.7)$ & $23(95.8)$ & $98(98.0)$ & $0.630(0.427)$ \\
Systolic dysfunction & & $1(4.2)$ & $8(8.0)$ & \\
Yes & $7(9.2)$ & $23(95.8)$ & $92(92.0)$ & \\
No & $69(90.8)$ & & $10(10.0)$ & $0.219(0.640)$ \\
Pericardial effusion & & $3(12.5)$ & $90(90.0)$ & \\
Yes & $7(9.2)$ & $21(87.5)$ & & \\
No & $69(90.8)$ & & \\
Pulmonary hypertension & & & \\
Yes & & & \\
No & & & \\
\hline
\end{tabular}

LVH=left ventricular hypertrophy.

\section{Discussion}

The prevalence of electrocardiographic (ECG) abnormalities in this study was high among both the HIVpositive patients on HAART and their HAART naïve counterpart. Nearly half of the study subjects had one or more ECG abnormalities. However, the HIV-HAART treated patients had a higher prevalence than the naive subjects. This high prevalence of ECG abnormalities is comparable but lower than that reported by Njoku et al [12] and Okoye et al [13] in studies done in South-Eastern, Nigeria where 73\% and $70 \%$ were reported among the HIV seropositive HAART naïve patients and a higher prevalence of $93 \%$ was reported by Njoku and colleagues in the HAART treated group.

Findings in this study was also similar to that reported in the SMART HIV study, a large prospective study where more than half of the study cohorts had minor to major ECG abnormalities with nearly 1 in 2 patients having minor ECG abnormalities and 1 in 13 patients with major ECG abnormalities.[14] Only patients with CD4 count $>350$ cells/mm3 were recruited in the SMART HIV study. Contrary to findings by Kabwe et al [15] and Okoye et al [11], ECG abnormalities in this study were reported more in the HAART treated cohorts than their naïve counterparts. Evidence suggests that lower cell CD4 counts are associated with elevated levels of activated CD4 $\mathrm{T}$ cells, this inflammatory cascade eventually leads to various forms of vascular and in turn myocardial damage [16] As a result many electrical abnormalities are prone to occur on ECG in PLWHIV/AIDS independent of the usual risk factors such as 
hypertension.

Sinus tachycardia was the most prevalent ECG finding in this study. Okoye et al reported a higher prevalence of sinus tachycardia of $64 \%$ [13]. Kabwe et al in a Zambian based study however reported significant bradycardia in about $7 \%$ of the study cohorts, there was however no reported case of bradycardia in this study [15] Njoku and colleagues also reported sinus tachycardia among the HAART naïve subjects [12] This high prevalence of sinus tachycardia in the treatment naïve arm could be explained by anxiety, excessive sympathetic stimulation from autonomic imbalance, dehydration in subjects with chronic diarrhea, anemia and increased metabolic demand in febrile illnesses as heart rate is known to increase with rise in body temperature [17].

The prevalence of LVH is comparable to that by Amusa et al [18] in Northern, Nigeria of $17.3 \%$ but lower than that reported by Kabwe and colleagues of $25 \%$ in a Zambian teaching hospital and this could be explained by the higher prevalence of hypertension among the study cohorts [15]. Left ventricular hypertrophy $(\mathrm{LVH})$ is defined as one of the major ECG abnormalities and has been found to be an independent predictor of cardiovascular mortality [19]

The prevalence of T-wave inversion was higher among the HAART treated group than the HAART naïve subjects $(23 \%$ vs $8 \%$ ) and these findings were similar to that reported in some local studies $[10,13]$. T-wave inversion signifies ischaemia but it's often a non-specific finding especially among women. Njoku et al however found a higher prevalence of $\mathrm{T}$ wave inversion of $47 \%$ among the HAART treated subjects compared to $30.4 \%$ in the naïve patients [12] ST-segment depression was reported in $2 \%$ of the HAART experienced patients. Non-specific ST and T wave changes on ECG have been reported in PLWHIV/AIDS caused by pericardial disease, myocardial disease or dilated cardiomyopathy $[20,21]$ Pathological Q waves was seen in one of the each of the case and control subject and this might be suggestive of an old myocardial infarction. Findings of such major ECG abnormalities was independently predictive of an incident cardiovascular event according to Soliman and colleagues in the SMART HIV study [14]

The prevalence of prolonged QT interval was 18\% among the cases versus $5 \%$ in the control arm and these were mostly minor QT prolongation. This prevalence was similar to that reported in the HIV-HEART study by Reinsch et al where a prevalence of about $20 \%$ was reported in HIV-infected individuals [22]. Factors like diabetes and arterial hypertension were however more prevalent in the HIVHEART study than in this study. Okoye et al however found hypocalcemia to be responsible for prolonged QT interval in his treatment naive subjects [11].

Other ECG abnormalities found in this study include conduction abnormalities like first degree A-V block, incomplete right bundle branch block and ventricular extrasystole. Left axis deviation and left atrial enlargement were also infrequently seen. Notable in this study was that asymptomatic ECG abnormalities were common in both HAART treated and HAART naïve patients with T-wave inversion and prolonged QT interval being more common in the HAART treated group and sinus tachycardia reported more in the HAART naïve group. Most of these findings were minor ECG abnormalities with a few major ECG abnormalities reported. When comparing the two HAART groups the ECG findings were still more prevalent in the non-PI based HAART group than the PI-based group and this could be explained by the fact that more than three quarter of the cases were on a non-PI based HAART regime compared to those on PI-based HAART.

This study reveals that majority of patients with HIV infection had asymptomatic echocardiographic abnormalities. These abnormalities were found both in the HAART treated and naïve patients, however some ECHO abnormalities were more common in the HAART naïve than in the treated arm. The prevalence of echocardiographic abnormalities in this study was $52 \%$ in the HAART treated group versus $60 \%$ in the HAART naïve group. This finding was similar to that reported by Olusegun-Joseph and colleagues in a tertiary institution-based study in Lagos, Nigeria where a prevalence of $78 \%$ was reported among the HIV positive patients as against $16 \%$ in the HIV-negative controls. [23]The prevalence of $\mathrm{LVH}$ was not significantly higher in the cases than the control subjects (31\% versus $29 \%)$, most other studies have reported a significant difference in the left ventricular mass index and chamber dimensions of HIV patients when compared to healthy controls and have found a significant difference $[22,24]$.

This study however found no significant difference in their mean left ventricular wall dimensions between both groups. Some authors have suggested that an increase in structural dimensions of the heart and LVMI in PLWHIV/AIDS may be related to subclinical atherosclerosis [25]. Kabwe et al in Zambia also found a similar prevalence of LVH of $23.4 \%$ among his study cohorts [13]. Mondy et al found a much lower prevalence of LVH of $6.5 \%$ and this could be attributed to the demographic characteristics of the study cohorts as only $29 \%$ of the total population were non-Hispanic blacks [25].

Cardiac dysfunction is prevalent in HIV patients. This study found the prevalence of LV diastolic dysfunction (LVDD) and LV systolic dysfunction (LVSD) among the HAART treated and naive patients to be $10 \%$ vs $23 \%$ and $2 \%$ vs $10 \%$ respectively with a higher prevalence in the naïve subjects. This finding has also been reported in several local and international studies. Owusu et al [26] in a teaching hospital-based study in Ghana reported an overall prevalence of diastolic dysfunction in $9.5 \%$, while LVSD accounted for $17.5 \%$.

A multicenter HIV-HEART study found a higher prevalence of systolic and diastolic dysfunction among to be $34.3 \%$ and $48 \%$ respectively. [21] Mondy et al in a prospective multisite study in the U.S also found a higher prevalence of LVDD than LVSD among their study cohorts, $26 \%$ versus $18 \%$ and this was similar to findings in this study [26]. Several local and international studies have found prevalence of LVDD among HIV-patients ranging from $9.5 \%-48 \%$ and LVSD ranging from $5.35 \%-34.3 \%$ and these 
findings were agreeable with that reported in this study [13$18]$.

The pathogenesis of HIV-associated diastolic dysfunction is likely multifactorial. Several studies have suggested that hypertension might be associated with prolonged duration of antiretroviral use. However, the prevalence of hypertension among study cohorts was low as previously known hypertensives were excluded. This factor alone was unlikely to account for our findings as we still found an association between HIV infection and diastolic dysfunction and this abnormality was mostly seen in the patients with a lower mean BP. HIV and other associated opportunistic infection may directly invade the myocardium causing myocarditis and subsequently diastolic and systolic dysfunction. HIV-1 has been detected in endomyocardial biopsy specimens in HIV patients with LV dysfunction [21] This may likely be the explanation why the untreated patients with a lower mean CD4 cell count had a higher prevalence of myocardial dysfunction than the patients on HAART. There was however no significant difference in myocardial dysfunction between the PI based and non-PI based group.

The prevalence of asymptomatic pericardial effusion in this study of $8 \%$ vs $11 \%$ among HAART treated and naïve patients was much lower than that reported by OlusegunJoseph et al in a teaching Hospital based study in Lagos, Nigeria where a high prevalence of $47 \%$ was reported.[23] This finding is contrary to that reported in the HIV-HEART study where a very low prevalence of $0.25 \%$ was reported and the risk of HIV associated pericardial disease was found to be increased with a lower CD4 cell [21] The HAART naïve patients with a lower mean CD4 also had a higher prevalence of pericardial disease in this study. Pericardial disease is said to be the most common cardiac manifestation of HIV infection [18] Studies done in Ilorin, Nigeria, Ghana, Zambia and the United States have reported prevalence varying from $0.25 \%-23.5 \%[10,13,22,23]$.

Pulmonary hypertension (PH) is an uncommon finding in HIV positive patients but the incidence is higher than that seen in the general population. Pulmonary hypertension has been associated with HIV induced chronic inflammation and endothelial proliferation. The prevalence of mild to moderate pulmonary hypertension in this study was $10 \%$ vs $5 \%$ among HAART treated patients and their naïve counterparts. The pulmonary valve acceleration time (PVAccT) was used to estimate mean pulmonary artery pressure (mPAP) as most subjects did not have tricuspid regurgitation. Kabwe et al [13] reported a prevalence of $3.7 \%$ also using PVAccT to estimate mPAP. Owusu et al [27] in Ghana however reported pulmonary hypertension as the most common ECHO finding among his cohorts with a high prevalence of $38.5 \%$ among the HIV positive treatment-naïve subjects but this was contrary to findings in this study where the HAART-treated subjects had a slightly higher prevalence. Mondy et al [26] also reported a prevalence of $57 \%$ among HAART-treated patients. This shows that the development of $\mathrm{PH}$ might be unrelated to treatment status as some patients with $\mathrm{PH}$ have other known risk factors such as chronic liver disease and intravenous usage [1].

Dilated cardiomyopathy was an uncommon finding in this study with a prevalence of $2 \%$ among HAART-naïve patients. The exact pathogenesis of DCM in HIV infection is unclear but myocarditis from direct invasion of the myocardium by HIV and co-infection with other viruses have been postulated [28]. A higher prevalence of $34 \%$ was however reported by Owusu et al among the treatment naïve patients [27]. The prevalence in this study was however similar but lower than that reported by Ogunmodede et al [24] of $2.7 \%$ and $5.05 \%$ by Olusegun-joseph et al [23] The development of DCM has been associated with untreated HIV infection and a low CD4 count and the prognosis of DCM in HIV infection is poor. [28] Since the advent of HAART for HIV infection, the clinical outcomes of PLWHIV/AIDS has improved substantially explaining the lower prevalence of ECHO abnormalities in the HAART treated patients.

\section{Conclusion}

Electrocardiographic and echocardiographic abnormalities are common in HIV seropositive patients with ECG abnormalities being more common among the HAARTtreated subjects. However, the higher prevalence of overt LV structural and functional dysfunction by ECHO among the HAART-naive patients suggests a relationship between an increase disease burden and the development of cardiovascular abnormalities. These ECG \& ECHO abnormalities generally increase the $\mathrm{CV}$ risk profile of PLWHIV/AIDS and are independent predictors of $\mathrm{CV}$ mortality, hence the need for prompt diagnosis and interventions. Electrocardiographic abnormalities were more common in the HAART-experienced patients with a significant difference in the prevalence of $T$ wave inversion and QT prolongation among the HAART-experienced patients when compared with their HAART-naïve counterparts. Sinus tachycardia was however the most significant finding among the HAART-naïve patients.

\section{References}

[1] Amado CL, Almeida AG. Cardiovascular disease associated with Human Immunodeficiency Virus: a review. Rev Port Cardiol. 2015; 34 (7-8): 479-491.

[2] Grinspoon SK, Grunfield C, Kotler DP, Currier JS, Lundgreen JD, Dube MP, et al. State of the science Conference Initiative to decrease cardiovascular risk and increase quality of care of patients living with HIV/AIDS: Executive summary. Circulation 2008; 118: 198-210.

[3] Lo J, Grinspoon S. Cardiovascular disease in HIV-infected patients: does HIV infection itself increase cardiovascular risk? Curr Opin HIV/AIDS. 2008; 3 (3): 207-213.

[4] Hescht SR, Berger M, VanTosh A, Croxson S. Unsuspected cardiac abnormalities in the Acquired Immunedeficiency Syndrome: an echocardiographic study. Chest 1989; 96: 805-808. 
[5] Reyskens PM, Essop MF. HIV protease inhibitors and onset of cardiovascular diseases: a central role for oxidative stress and dysregulation of the ubiquitin-proteasome system. Biochims Biophys Acta 2014; 1842 (2): 256-268.

[6] Stein JH, Klein AM, Bellehumeur JL, McBride PE, Wiebe DA, Otvos JD et al. Use of Human Immunodeficiency Virus-1 Protease Inhibitors Is Associated with atherogenic lipoprotein changes and endothelial dysfunction. Circulation 2001; 104: 257-262.

[7] Krikke M, Hoogeveen RC, Hoepelman AIM, Visseren FLJ, Arends JE. Cardiovascular risk prediction in HIV-infected patients: comparing the Framingham, atherosclerotic cardiovascular disease risk score (ASCVD) Systemic Coronary Risk Evaluation for Netherlands (SCORE-NL) and Data Collection on Adverse Events of Anti-HIV Drugs (D: A: D) risk prediction models. HIV Medicine 2016; 17: 289-297.

[8] Lang RM, Bierig M, Devereux RB, FlachKampf FA, Foster E, Pelikka PA et al. Recommendations for chamber quantification: a report from the American Society of Echocardiography guidelines and standards committee and the chamber quantification writing group, developed in conjunction with the European Association of Echocardiography. J Am Soc Echocardiogr. 2005; 18 (12): 1440-1463.

[9] Teichholz, LE, Kreulen TH, Herman, MV, Gorlin R. Problems in echocardiographic volume determinations: Echocardiographic-angiographic correlation in the presence of absence of asy-nergy. American Journal of Cardiology. 1976; 37 (1): 7-11.

[10] Nagueh SF, Smiseth OA, Appleton CP, Bryd BF, Dokainish $\mathrm{H}$, Edverdsen $\mathrm{T}$ et al. Recommendations for the evaluation of left ventricular diastolic function by Echocardiography. An update from the American Society of Echocardiography and European Association of Cardiovascular Imaging. J Am Soc Echocardiogr 2016; 29: 277-314.

[11] Ojikutu RK. The prevalence of cardiovascular diseases in Lagos state, Nigeria. Ghana Journal of Developmental Studies. 2009: 6 (2): 41-50.

[12] Njoku PO, Ejim EC, Anisiuba BC, Ike SO, Onwubere BJC. Electrocardiographic findings in a cross-sectional study of Human Immunodeficiency Virus (HIV) patients in Enugu, South-East Nigeria. Cardiovasc J Afr 2016: 27: 252-257.

[13] Okoye IC, Anyabolu NE. Electrocardiographic abnormalities among treatment-naïve HIV subjects in South-East Nigeria. Cardiovasc J Afr 2017; 28.

[14] Soliman EZ, Prineas RJ, Roediger MP, Duprez DA, Boccara F, Boesecke $\mathrm{C}$ et al. Prevalence and prognostic significance of ECG abnormalities in HIV-infected patients: results from the Strategies for Management of Antiretroviral Therapy study. J Electrocard 2010; 44 (6) 779-785.

[15] Kabwe L, Lakhi S, Kalinichenko S, Mulenga L. Prevalence of subclinical cardiovascular disease in healthy HIV infected patients at the University Teaching Hospital in Lusaka, Zambia. Medical Journal of Zambia, 2016; 43 (1): 12-23.

[16] Robbs JV, Paruk NManagement of HIV vasculopathy-a South African experience. Eur J Vasc Endovasc Surg. 2010 Mar; 39 Suppl 1: S25-S31.

[17] Karjalainen J, Viitasalo M. Fever and cardiac rhythm. Arch Intern Med. 1986; 146 (6): 1169-1171.

[18] Amusa GA, Akanbi MO, Okeahialam BN, Danbauchi SS, Onuh JA, Uguru SU et al. Electrocardiographic Abnormalities in HIV-infected Adults in North-Central Nigeria. Am J Respir Crit Care Med 2016; 193: A7050.

[19] Havranek EP, Froshaug DB, Emserman CDB, Hanratty R, Krantz MJ, Masoudi FA et al. Left Ventricular Hypertrophy and Cardiovascular Mortality by Race and Ethnicity. Am J Med. 2008; 121 (10): 870-875.

[20] Ntsekhe M, Mayosi BM. Cardiac manifestations of HIV infection: An African perspective. Nature Clinical Practice Cardiovascular Medicine. 2009; 6 (2): 120-127.

[21] Rerkpattanapipat P, Wongpraparut N, Jacobs LE, Kotler MN. Cardiac manifestations of Acquired Immunodeficiency Syndrome. Arch Intern Med. 2000; 160 (5): 602-608.

[22] Reinsch N, Kahlert P, Esser S, Sundermeyer A, Neuhaus K Brockmeyer $\mathrm{N}$ et al. Echocardiographic findings and abnormalities in HIV-infected patients: results from a large, prospective multicenter HIV-HEART study Am J Cardiovasc Dis. $2011 ; 1$ (2): 176-184.

[23] Olusegun-Joseph DA, Ajuluchukwu JNA, Okany CC, Mbakwem AC, DA Oke DA, Okubadejo NU et al. Echocardiographic patterns in treatment-naïve HIV-positive patients in Lagos, South-West Nigeria. Cardiovasc J Afr 2012; 23: e1-e6.

[24] Ogunmodede JA, Kolo PM, Katibi IA, Salami AK, Omotoso A. Structural echocardiographic abnormalities seen in HIV/AIDS patients are independent of CD4 count. Niger J Clin Pract 2017; 20: 716-723.

[25] Hsue PY, Hunt PW, Ho JR, Farah HH, Schnell A, Hoh R et al. Impact of HIV infection on diastolic function and left ventricular mass. Circ Heart Fail 2010; 3: 132-139.

[26] Mondy K, Overton ET, Grubb J, Tong S, Sey-fried W, Powderly $\mathrm{W}$ et al. Metabolic syndrome in HIV-infected patients from an urban, Midwestern US outpatient population. Clin Infect Dis. 2007; 44 (5): 726-734.

[27] Owusu KI, Oppong B. Echocardiographic abnormalities in patients with HIV Infection at Komfo Anokye Teaching Hospital, Ghana J Gen Pract 2014; 2: 2.

[28] Herskowitz A, Vlahos D, Willoughby S, Chaisson RE, Schulman SP. Prevalence and incidence of left ventricular dysfunction in patients with Human Immunodeficiency Virus infection. American Journal of Cardiology. 1993; 71 (11): 955-958. 